\title{
Can we expect the massive discs around young stellar objects class O-I to be a birthplace of planetesimal?
}

\author{
Valeriy N. Snytnikov ${ }^{1,2}$, Olga P. Stoyanovskaya ${ }^{1,2}$ \\ and Olga A. Stadnichenko ${ }^{1,2}$ \\ ${ }^{1}$ Boreskov Institute of Catalysis SB RAS \\ Pr. Academika Lavrentieva, 5, 630090, Novosibirsk, Russia \\ email: snyt@catalysis.ru, stop@catalysis.ru, zasypoa@catalysis.ru \\ ${ }^{2}$ Novosibirsk State University, \\ Str. Pirogova, 2, 630090, Novosibirsk, Russia
}

\begin{abstract}
Possibility of large bodies formation in massive discs of young stellar objects (YSO) class O-I was investigated. On the stage of YSO O-I the whole of factors: chemical composition of gas and solids, chemical catalytic reactions, the disc self-gravitation, the increased ratio of solids to gas surface density, adiabatic gas cooling provides favorable conditions for gravitational instabilities development. We simulated 3D dynamics of gas and dust under self-consistent gravitational field and reproduced the formation and evolution of the disc around the protostar. We found that for stars of Solar mass there are regimes when the disc of variable mass is unstable for the development of fast gravitational instabilities.
\end{abstract}

Keywords. Young Stellar Object (YSO) class O-I, gravitational instability, self-gravitating clump formation.

Star and planetary system formation goes via fragmentation of a molecular cloud with gravitational collapse of a solitary clump into protostar with a circumstellar accretion disc, transformation of protostar into star, gas loss and appearance of planets and other bodies in the disc. This evolutionary sequence is distinguished as young stellar object (YSO) of class 0, I, II, III. The early phase of young stellar objects (class 0 and I) is the most difficult for observation. In such objects the protostar is surrounded by a disc of comparable mass. On this stage fast luminosity changes are recorded for some objects, which indicated that they are unstable. Gravitational instability of such objects can be responsible for planetary formation processes as well as the protostar mass increasing.

In this paper we list condition (1)-(4) for massive circumstellar disc of YSO of classes 0 and I that provides the development of gravitational instability over timescale of several orbital periods. Mass and temperature distribution for accretion disc is determined by the infall of opposing gas-dust streams and their collision. Then the gas moves along the rotation axis away from the equatorial plane. The gas expansion decreases its temperature and leaves the coagulating dust in the plane. Over the time 0.5 million year (1) the mass of the disc reaches the mass of the star (Snytnikov et al. (2013)), and then the disc mass decreases by the million year to 0.1 of star mass. In the circumstellar disc the the subdisc made of solid bodies form. (2) In the subdisc the ratio of solid to gas concentration is increased with respect to the molecular cloud. The composition of the solid is defined by the cosmic abundance of elements, according to which the fraction of water and organic compounds is higher than the fraction of inorganic substance (Herbst \& van Dishoeck (2009)). On the stage of YSO of classes 0 and I coagulation and aggregation of such solids (when the evaporation and decomposition of organic has not led already to prevalence of 
inorganics) may lead to (3) the formation of metre-sized bodies. Gas drag decreases the velocity dispersion of such solids and causes their infall onto the protostar over a timescale of 0.1 Myr. Chemical reaction such as, for instance, synthesis of molecular from atomic hydrogen on the surface of solids, can affect (4) the effective adiabatic exponent.

To simulate three-dimensional dynamics of a gas cloud, we used a numerical code developed by the authors (Snytnikov \& Stadnichenko (2011)). The code is based on the FLIC method and employs a uniform mesh in Cartesian coordinates. The method provides the first order approximation over space and time. The gas dynamics code solves the initial-boundary value problem for non-viscous gas in a closed region with the initial conditions coinciding with the Bonnor Ebert solution. The boundary conditions ensure the absence of shocks on the boundary at nonzero mass flow. The calculations demonstrate the development of physical gravitational instability in the formed gaseous disc.

We carry out numerical simulation of multiphase massive disc dynamics with the development of global gravitational instability. We found that local gravitational collapse which can form big planetesimal or gas giant can be an outcome of the instability development over a timescale of several orbital period (Snytnikov \& Stoyanovskaya (2013)).

This work was supported by RFBR project 14-01-31516, the RAS Presidium programs 'Biosphere origin and evolution' and 'Origin, structure and evolution of objects in the Universe', the SB RAS Integration Project N130. The work was also supported by the Ministry of Education and Science of the Russian Federation.

\section{References}

Snytnikov, V. N. \& Stoyanovskaya O. P., 2013, MNRAS, 428, 2

Snytnikov, V. N., Stoyanovskaya, O. P., \& Stadnichenko, O. A., 2013, EPJ Web of Conferences, 46, 07004

Snytnikov, V. N. \& Stadnichenko, O. A., 2011, Astron. Rep., 55, 214.

Herbst, E. \& van Dishoeck, E. F., 2009, Ann. Rev. Astron. and Astrophys., 47, 427 